\title{
Spontaneous Left Main Coronary Artery Dissection: Case Report
}

\author{
S. V. V. Mani Krishna ${ }^{1}$ \\ D. P. Kalyan Chakravarthy ${ }^{1}$ \\ Shahood Ajaz Kakroo ${ }^{1}$ \\ M. Naveen Kumar ${ }^{1}$
}

${ }^{1}$ Department of Cardiology, NIMS, Punjagutta, Hyderabad, India

Address for correspondence S. V. V. Mani Krishna, DM, Department of Cardiology, NIMS, Punjagutta, Hyderabad 500082, India (e-mail: manikrishnas009@gmail.com).

Ind J Car Dis Wom 2021;6:39-41.

\author{
Abstract \\ Keywords \\ - spontaneous left \\ main coronary artery \\ dissection \\ - spontaneous \\ resolution \\ - conservative \\ management
}

Left main coronary artery (LMCA) dissections may be iatrogenic or spontaneous. Spontaneous coronary artery dissections (SCADs) are common in pregnant women, with predominantly left anterior descending artery (LAD) involvement. In males, coronary artery dissections may happen in alcoholics. Spontaneous LMCA dissection as the cause of myocardial infarction is very rare. We are reporting a case of spontaneous LMCA dissection in a 45-year-old male alcoholic with the involvement of LAD ostium, which resolved spontaneously with conservative management.

\section{Introduction}

Spontaneous coronary artery dissection (SCAD) is an infrequent cause of ischemic heart disease, resulting in sudden death. ${ }^{1}$ This category, the spontaneous coronary dissection of the left main coronary artery (LMCA), is decidedly less common, accounting for $6-12 \%$ of all primary coronary dissections. ${ }^{1}$ Usually, this leads to sudden death or extensive myocardial infarction. Treatment modalities for SCAD include conservative medical management, percutaneous coronary intervention (PCI), and coronary artery bypass graft surgery (CABG) surgery. For this disease process, the optimal treatment strategy has still not been clearly defined. We are reporting a case of spontaneous LMCA dissection.

\section{Case Details}

A 45-year-old male, who was hypertensive alcoholic, came to our institution with 16 hours of chest pain and mild pain at the time of admission. He had a sudden onset of severe chest pain, squeezing in nature while performing heavy work on his farm. There was neither any radiation of the pain

published online April 9, 2021
DOI https://doi.org/ 10.1055/s-0041-1723919. to the back, nor was it associated with breathlessness. The patient was hemodynamically stable with normal volume of all peripheral pulses. ECG taken outside hospital showed ST elevations in II, III, AVF and V3 V4 V5 V6 (-Fig. 1). ECG done in our center showed symmetrical $\mathrm{T}$ wave inversion from V1-V6 along with Q waves in II III AVF.

Two-dimensional (2D) echo showed mild hypokinesia of distal intraventricular septum (IVS), apex and mild left ventricular (LV) dysfunction. As per the 2D echo, there is no evidence of aortic dissection. The patient was taken up for coronary angiography. Coronary angiography (CAG) showed a regular luminal filling defect in the anterosuperior aspect of the LMCA. There is a free mobile distal part, which is prolapsing into left anterior descending (LAD) artery and left circumflex (LCX) artery in different phases of the cardiac cycle ( - Fig. 2). The differential diagnosis of LMCA freely mobile thrombus versus dissection was considered. If it were considered a mobile thrombus, then even before the thrombuster insertion for thrombus aspiration, or the wire passage time itself, there would be higher chances of embolization. So, it was decided to manage the lesion with tirofiban infusion for 24 hours pharmacologically. Thrombolysis was not 


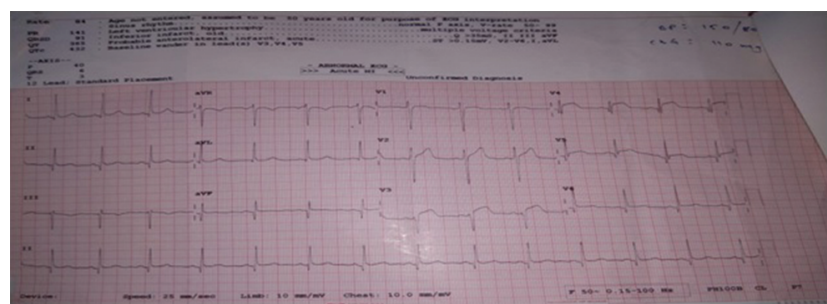

Fig. 1 ECG showing ST elevation in V3 V4 V5 V6 and Q waves in II III AVF.

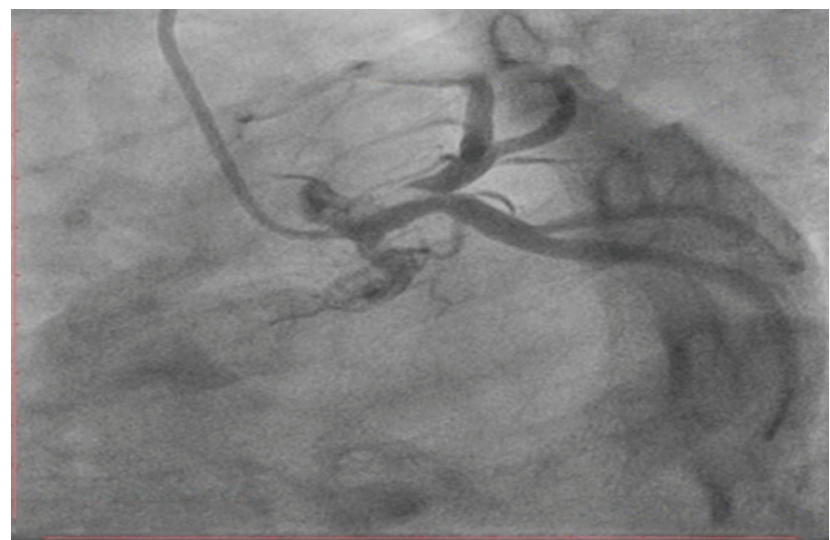

Fig. 2 Initial angiogram showing luminal lucency with finger like projection in the left main coronary artery (LMCA) anterosuperior aspect.

considered as the patient was out of the window period (16 hours). It might have predisposed the patient for cardiac rupture. CAG was done on the following day and showed the same lesion.

After considering the possibility of LMCA dissection more likely, to delineate the LMCA lesion more accurately and rule out aortic dissection, CT CAG and CT aortogram were performed. CT CAG showed a thin intimal flap along the anterosuperior wall of LMCA, extending into the ostium of LAD, with false lumen up to LAD ostium and prominent pericoronary fat haziness ( - Fig. $\mathbf{3} \mathbf{a}, \mathbf{b}, \mathbf{c}$ and $\mathbf{d}$ ). As per the radiologist, the flap was very thin and the false lumen very small, but it was a healing flap.

The patient remained asymptomatic, but as the LMCA dissection had culminated in a myocardial infarction, LMCA stenting under optical coherence tomography (OCT) guidance was planned. On the day of elective stenting, that is, 4 days after the first CAG, a normal LMCA showed, which is suggestive of spontaneous closure of the dissection with conservative management, a rare occurrence ( - Fig. 4 ).

\section{Discussion}

Most of the primary coronary dissections occur in the third or fourth decade of life in peripartum (75\%-LAD, 20\%-right coronary artery (RCA), 4\%-LCX, 1\%-LMCA) . Even though the cause is idiopathic in most of the cases, spontaneous coronary dissections are also associated with trauma, angiitis, sarcoidosis, Marfan's syndrome, previous renal transplantation,
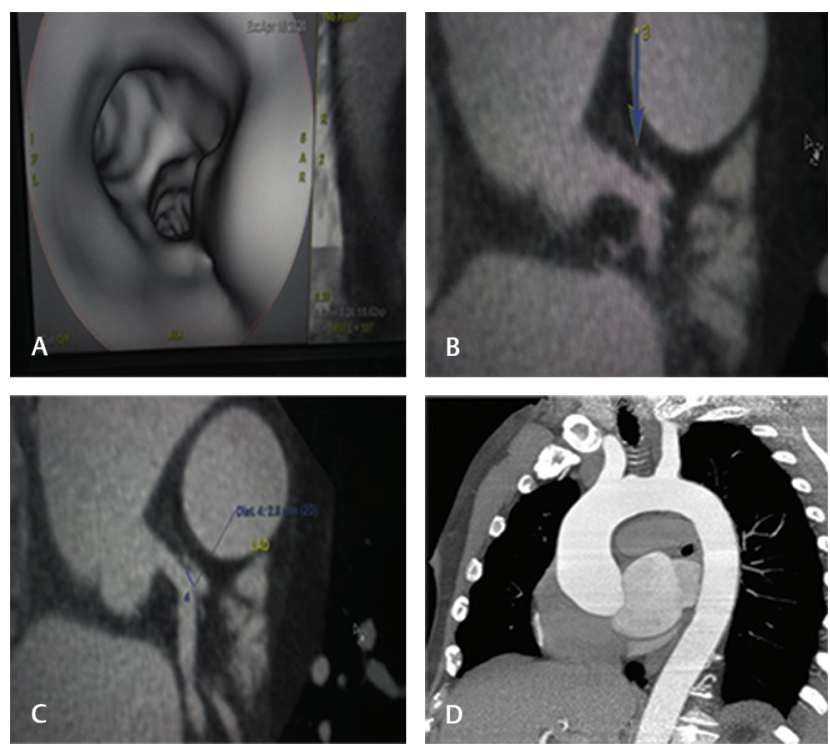

Fig. 3 (A) CT coronary angiography (CAG) intravascular ultrasound (IVUS) view of left main coronary artery (LMCA) with bifurcation showing lesion along the anterosuperior wall with extension to left anterior descending (LAD) artery ostium. (B) CT CAG showing aortic root along with LMCA showing dissection flap (blue arrow) as radiopaque line with free hanging edge at the distal LMCA. (C) CT CAC showing dissection flap extending into LAD ostium. (D) CT aortogram showing normal study.

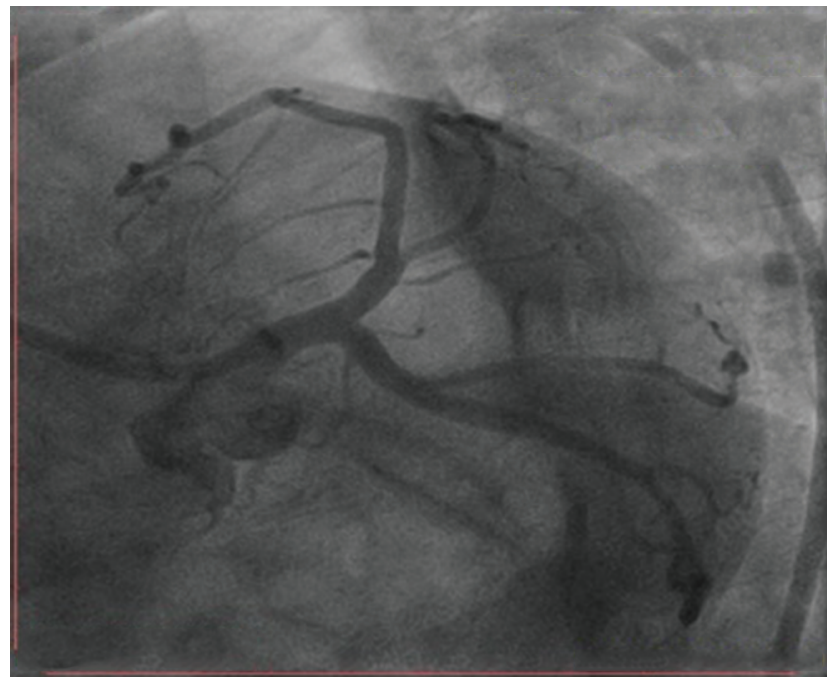

Fig. 4 Final coronary angiogram showing normal left main coronary artery (LMCA) suggestive of closure of dissection.

heavy exercise, prolonged sneezing, alcoholism, and use of cocaine, cyclosporine and oral contraceptives. ${ }^{2}$ Hypertension is not a risk factor for SCAD.

Although all these conditions are usually associated with the weakening of connective tissue and vascular inflammation, SCAD's exact cause and pathogenesis are not established. In both men and women, pathological analysis of postmortem spontaneous left main coronary dissections frequently reveal vascular adventitia rich in eosinophils, which suggests that arterial wall damage is due to the result of release of lytic substances from these cells. Moreover, it is 
uncertain if the local inflammatory cell infiltrate is causal or reactive to the process. ${ }^{3}$

The clinical spectrum of SCAD also differs according to gender, as LAD is more frequently involved in women. ${ }^{4}$ In men, RCA is more often involved, and clinical presentation occurs later in life. However, in our case, dissection involved the LMCA. There are often associated atherosclerotic coronary artery disease and cardiovascular risk factors seen in men; as in our case it was hypertension and alcohol intake.

The patient's clinical condition, location and extent of dissection, and its functional consequences, will decide the choice of initial management. If there is no evidence of ongoing ischemia or hemodynamic instability, SCAD can be managed successfully with conservative management, especially in cases of single vessel dissection not affecting the LMCA with thrombolysis in myocardial infarction (TIMI) 3 flow. ${ }^{5}$ Medical management includes antithrombotic therapy with heparin or low-molecular weight heparin, aspirin, clopidogrel and glycoprotein IIb/IIIa inhibitors, and anti-ischemic therapy with $\beta$-blockers and nitrates. However, it is also possible that while potent antithrombotic therapy decreases thrombus formation in the false lumen and enhances blood flow in the true lumen, it can also increase bleeding into the false lumen, causing extension of the dissection. It is more common with the use of fibrinolytics, and they should be avoided. ${ }^{6,7}$ That is the other reason why we preferred tirofiban infusion over fibrinolysis.

Conservative management is a reasonable approach in asymptomatic, stable patients with distal coronary artery dissection or preserved coronary blood flow. In our case, the dissection started from the ostium. Fortunately, it did not involve the ascending aorta. The subsequent course was stable and asymptomatic, with TIMI 3 flow from the beginning. It encouraged us to go ahead with conservative management. If more aggressive therapy was planned during the initial stages, in which imaging becomes mandatory to distinguish from mobile thrombus, it might have resulted in extension of the dissection, as the initial dissection was from the ostium of LMCA. Initially, giving tirofiban infusion also might have been an appropriate therapy. If it had been coronary thrombus, tirofiban would have aided in dissolving and preventing the extension of thrombus and, in the case, would have been a coronary artery dissection, to prevent the thrombus in the false lumen (with minor risk of dissection extension or hemorrhage in the false lumen). CT CAG also helped in risk stratifying this case, as partial dissection of healing and no dissection or any pathology in the aorta was demonstrated. Therefore, we could exclude predominant connective tissue disorders and manage medically.

\section{Conclusion}

Differential diagnosis between SCAD and coronary thrombus is not always straight forward on angiography, so the use of intravascular ultrasound (IVUS), OCT and CT CAG is needed for correct diagnosis. If the patient is stable, for LMCA dissections, CABG or PCI may not always be needed, as spontaneous closure can occur.

Conflicts of Interest

None declared.

\section{References}

1 Vrints CJM. Spontaneous coronary artery dissection. Heart 2010;96(10):801-808

2 McCann AB, Whitbourn RJ. Spontaneous coronary artery dissection: a review of the etiology and available treatment options. Heart Vessels 2009;24(6):463-465

3 Siegel RJ, Koponen M. Spontaneous coronary artery dissection causing sudden death. Mechanical arterial failure or primary vasculitis? Arch Pathol Lab Med 1994;118(2):196-198

4 Mortensen $\mathrm{KH}$, Thuesen L, Kristensen IB, Christiansen EH. Spontaneous coronary artery dissection: a Western Denmark Heart Registry study. Catheter Cardiovasc Interv 2009;74(5):710-717

5 Lemos-Neto PA, Ramirez JA, Sposito AC, et al; da Gama MN. Spontaneous healing of primary dissection of the coronary artery. J Invasive Cardiol 1999;11(1):21-24

6 Le MQ Ling FS. Spontaneous dissection of the left main coronary artery treated with percutaneous coronary stenting. J Invasive Cardiol 2007;19(8):E218-E221

7 Saw J, Starovoytov A, Humphries K, et al. Canadian spontaneous coronary artery dissection cohort study: in-hospital and 30-day outcomes. Eur Heart J 2019;40(15):1188-1197 